\title{
Research on the Integration and Performance of Emotion Concept in Visual Communication Design
}

\author{
Huifang Li and Qi Wang \\ Hebei Academy of Fine Arts, Shijiazhuang, Hebei Province, 050700, China
}

Keywords: Visual Communication Design, Visual Emotion, Constituent Elements

\begin{abstract}
Visual communication design is an important part of modern design, which is the main form of information communication in modern society. The existing design is no longer only satisfied with the function and the surface of the visual experience, but to consumers and viewers through visual language to convey a certain emotional experience. This paper mainly expounds the elements of visual communication design, in order to provide a reference for the designer.
\end{abstract}

\section{Introduction}

Man is the animal of the feeling, the effective emotional expression is an important part of human nature. With the rapid development of modern social economy, science and technology, it is lack of affection between people, the emotional indifference, at a distance, these phenomena have attracted a lot of relevant experts and scholars attention, As a designer [1], face and think about is how to design works with the humanity and emotion, and how to make people while accept transfer product design information and its emotional communication. So just as to obtain the emotions and various emotions, the increasingly indifference and coldness of social relations were need to ease [2]. So the role of emotional design in the future design is becoming increasingly important. [3].

Emotional expression, is a kind of design for the design of the new performance of human nature [4]. Human civilization has been able to continue to develop and prosper, is to meet the needs of the continuous improvement of human results, design is also so, the need for human beings, and to express this is mainly reflected by the emotions, and the core of the creative process, the designer is "emotional". From the visual communication design of the most close to the daily life of the creative form of the emotional concept of mining and performance, and then the various disciplines of mutual contact and mutual penetration, visual language, graphics, creative, color collocation, text layout will be more widely used in the design environment, designers are increasingly aware of the importance of the design of emotional expression, are beginning to pay attention to find the emotional expression of emotion [5].

Because of the continuous pursuit of life quality, people's need is update to chase more convenient, more comfortable living things. But the reality is not satisfied with the attitude, and the desire to achieve self-value. So that the existing design works continuous improvement in function, beauty, emotional and other aspects, in order to meet people's requirements better. Emotional design is to grasp the characteristics of human emotion, for the audience, in order to design the performance approach, to meet their needs. Emotional concept in the design of the performance reflects the respect and care for people, but also the forefront of the trend [6].

Xi'an Jiao Tong University and University of Science and Technology Beijing published a paper on the design of the related work, and to carry out the relevant research on Sino Japanese cooperation[7]; Shanghai Jiao Tong University professor Zhang Xianrong published in 2001, the feeling of the measurement, in the domestic research has focused on the academic value of the study, so that the study of the qualitative research into quantitative research. It is reported that since 2003, China, Japan and South Korea have begun to jointly carry out the study of the relevant issues, which is a good start for the Chinese design community.

In our country, the concept of emotion is reflected in the design of product design, film and television media animation, industrial design and other fields, the visual communication design field of emotion concept is not obvious[8].

Foreign related design field of two major research: Japan's emotional engineering and Donald 
-A-Norman( December 25, 1935 -) three levels of emotional design[9].

Japanese Kansei (Engineering): Professor at Hiroshima University, Professor of the, the emotional engineering is a kind of customer oriented development technology, a customer's experience and intention to translate into the design elements of translation technology [10]. The first proposed the term "Kansei Engineering" is Japan Toyo kogyo KK group (now Mazda Motor Group), former president of the yamaken Gumi, 1986 he by the University of Michigan in the United States a "car culture on" speech, put forward the concept of, and caused great repercussions.

\section{Emotional Performance in Visual Communication Design}

Emotional Expression and Design Work. Emotion has a great influence on people's thinking, people's mood. And emotion is an important part of human mental activity, if people have no mood or emotion, it will lead to a certain obstacle to their thinking ability. In the process of visual communication, the only real injection of emotion, is reflecting the emotions. It can give different works of meaning and vitality, increase its depth, so that the work can be moved. And if faucet works without components of emotion in sight. It is very easy to cause the design works have a shell, is likely to cause everybody's repugnance, can not reflect the works of true value. As show in figure 1

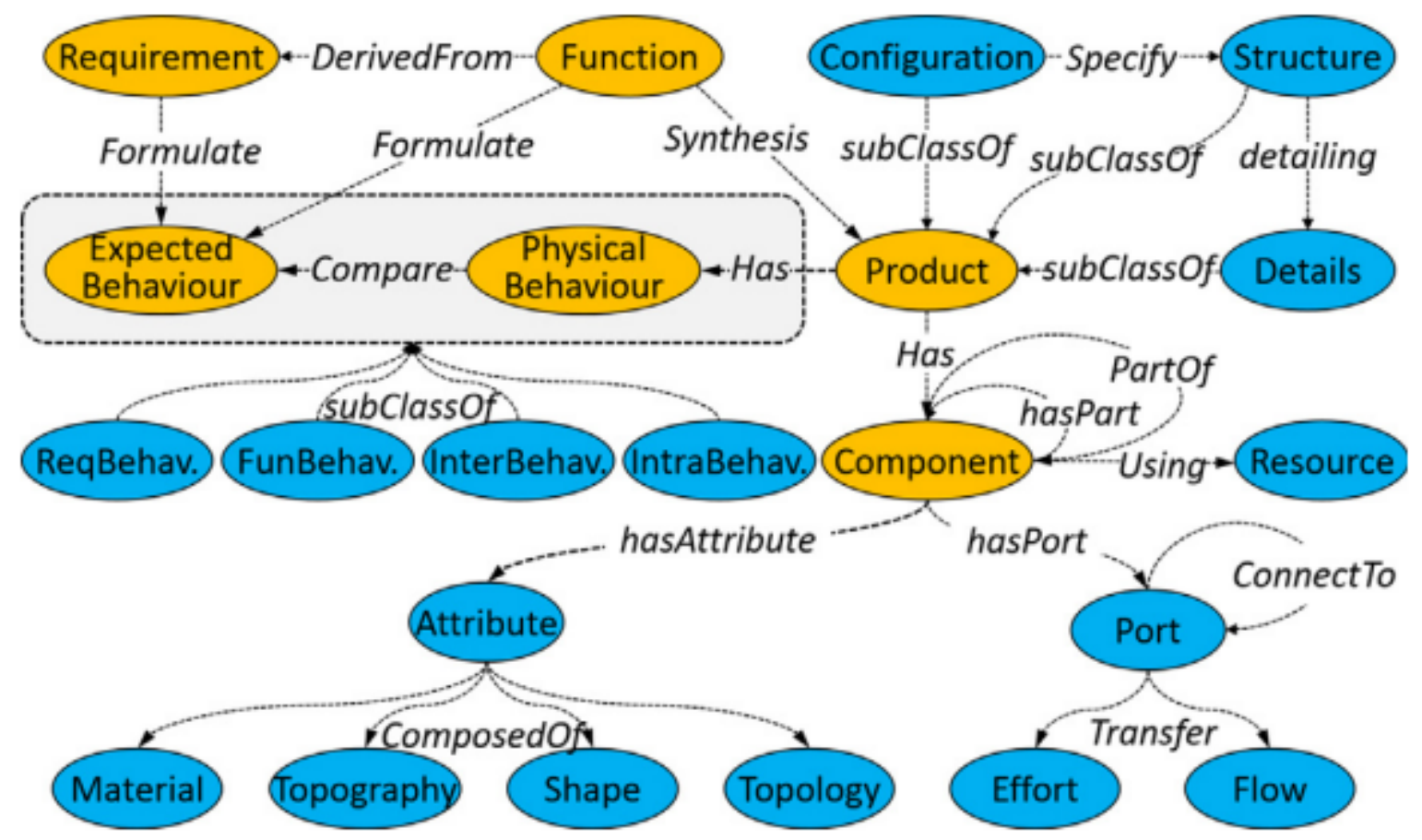

Figure 1. Ontology of automating design and its semantic-network

Emotional Performance and Visual Communication Design Ideas. Emotional performance is usually refers to the design idea of a kind of human performance, usually in the case, designers are required to have a certain emotional performance. Humanized design idea is the theme of the design work under the background of the new era. The work not only can play a certain role in the human spirit, but also can affect the state of human mind.

\section{Form and Design Emotion Performance}

As one of the main components of visual communication, the form is a relatively superficial element, with obvious intuition. It can be divided into points, lines and surfaces. As show in figure2. 


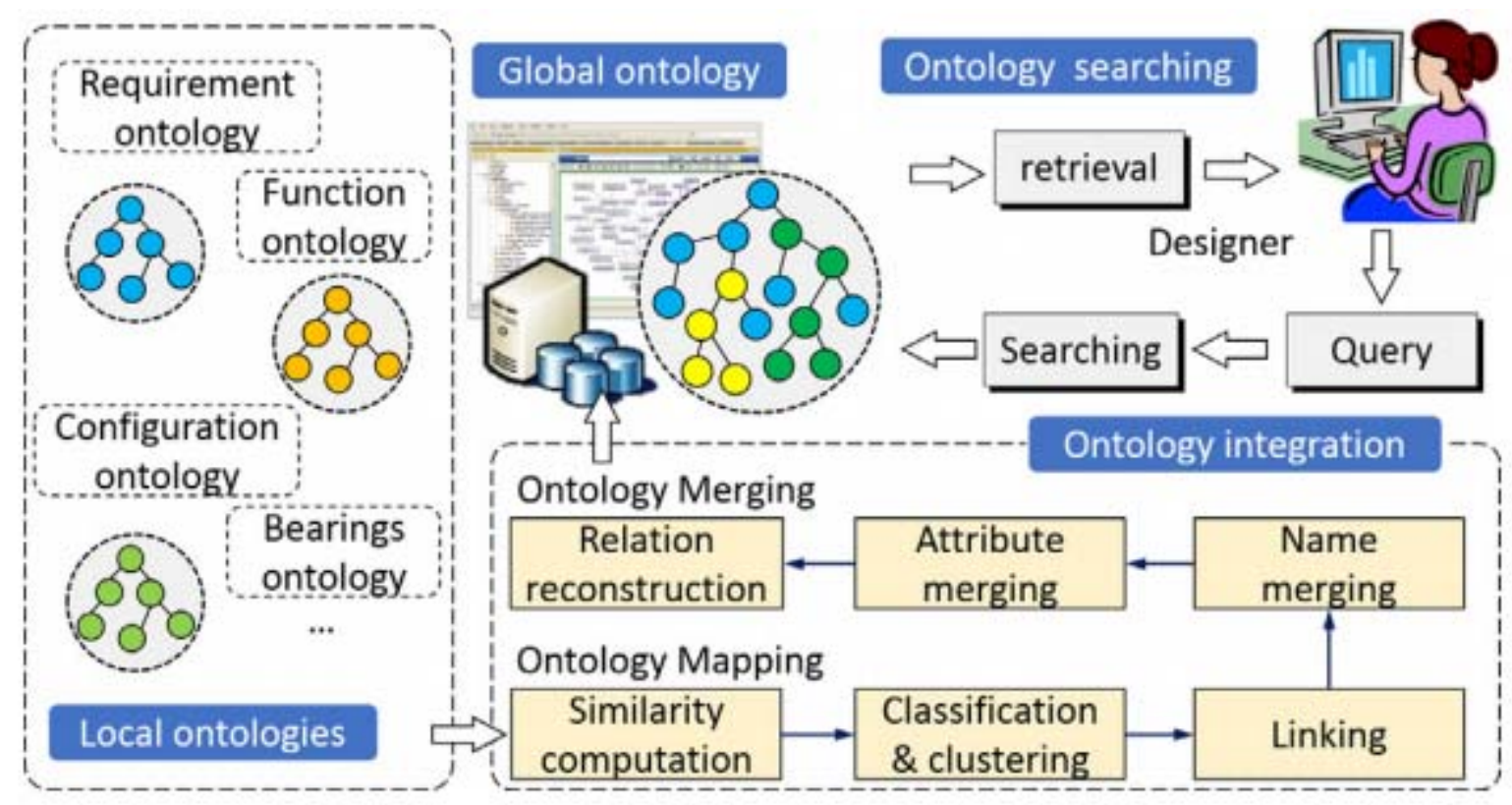

Figure 2. Pyramid of semantic web languages

On the "Point" of the Emotional Performance. Knowledge of geometry, the point is the element of the size of the non-direction, which simply represents a position. However, in visual communication design group, the elements also have differences in size and shape and position on the different part exist shades of difference, there is virtual, are scattered together. For the design of visual communication design, the definition of the point is closely related to human visual perception.

On the Emotional Expression of the "Line". In the process of comprehensive analysis of the external environment, the first step is to observe the shape and contour of the object, forming a preliminary understanding, the line is a basic element in the whole form and the outline, can play a decisive role in the emotional expression of the whole work. In general, a straight line creates a simple, plain feeling, a balance of feeling, and a curve that represents a soft, mellow feeling. Designers can use the different emotional attributes of lines, the main idea of the design of the main body, the objective of the food and the audience to establish an effective contact, so as to give full play to the impact of the design work on the audience.

On the Emotional Expression of "Facial". Surface in the whole visual form of an important position, the element is much more fill than the point and line, usually can be divided into two types, one is the plane, the other is curved surface. For visual communication design, usually based on the plane. Plane can be divided into three different types, the first one is geometric shape, the second is an organic form, the third is not a rule. The first type of surface can create a kind of Shu Lang, simple visual feelings. However, this kind of plane is also very easy to let the audience feel boring and old-fashioned. Organic form is usually extracted from natural things, plant leaves, clouds and other organic matter, which can create a kind of natural kind of visual experience, and makes people feel very well.

\section{Emotional Expression in Visual Communication}

Modeling emotion is usually caused by the macro and micro factors, which may be a kind of inherent emotional attributes, and it may be a component of the work design . Design personnel in the visual communication design process, it should be standing in the macro perspective and micro perspective, for the work of the modeling approach to scientific design, as far as possible to ensure that the main body of the work adapt to the main ideas. 


\section{Emotional Expression of Color Elements in Visual Communication}

Color in human visual perception system has obvious intuitive, this element has a very close relationship with human factor and emotional factors, it also can play an important role in the whole design idea. Design personnel must be comprehensive analysis of the brightness and purity of color, and the specific study of its changes in temperature and depth, and so on. For the color of the practical application of the method and the implementation of specific analysis and research, the use of scientific methods to promote the emotional effect of good play. In the concrete operation, according to the view of color psychology, red from the positive point of view to consider with passion and energy, and in the negative point of view, showing a kind of emotional performance, such as anxiety and danger.

\section{Emotional Expression of Material Texture in Visual Communication}

All objective things have the corresponding texture. The texture can be scientifically demonstrated in the form of things, improve the sense of the image and the sense of hierarchy, and play a good role in the picture to achieve the purpose of creating a visual sense of beauty. At the same time, muscle strength has a certain symbolic meaning, can help human beings to fully express the emotion of the picture. Under normal circumstances, the wood texture is a natural and kind of feeling, and the metal texture is generally more rational, with a sense of science and technology as well as avant-garde sense [18]. Glass texture can project a kind of elegant, fine and lively visual experience; the leather texture has the characteristics of sensibility and warmth; stone texture is relatively quiet, can give a person a kind of emotional performance, and have a certain human color.

\section{Test Results}

In a word, emotion is a great influence on people's thinking, people's mood and emotion are important part of human mental activity, if people lack of interest or emotion, it will lead to a certain obstacle to their thinking ability. In the process of visual communication, the only real injection of emotion, reflecting the emotions, can give different works of meaning and vitality, increase its depth, so that the work can be moved. Emotional performance for the entire visual communication design is very necessary and important, all designers must be based on the emotional performance, to adhere to the emotional as a design source, comprehensive analysis of the shape, shape and color and other aspects of the work of visual communication design, rich and full of the connotation of the work, to facilitate the work and the hands to achieve visual communication and emotional communication, to achieve the design of human nature.

\section{Conclusion}

I believe that the emotional design is not a simple passion of the designer, and should be born in the user's psychological. The real emotional design should be "generated in deep knowledge and strive to achieve a certain kind of psychological interaction with consumers", good design should make use of this design of people feel the product and the distance is very close, and can be connected with people's heart. From the point of view, the product is influenced by the emotional factors as the breakthrough point, and the emotional reaction is influenced by what way people used. Mainly through the analysis of the form, color and material of the design from the perspective of semiotics and cognitive psychology, as well as the way of how to influence the mood of the audience. The symbolic meaning of the high-tech products is a common one in the world, and it is easy to get the sympathy from the audience. And because of the natural yearning for love, can reflect the beauty of the natural the design will always be able to meet the emotional needs of specific consumer. In general, the emotional design is to be able to pursue from a rational angle degree to meet the people's emotional needs, and in a reasonable and harmonious means to show. 
(1) Combining the concept of experience economy and experience design, this paper analyzes the differences of the relationship between the design and the consumer in the form of experience economy, and expounds the concept, nature and characteristics of product experience design. To sum up the understanding of "emotion" in different subjects, to propose and discuss the emotional experience is the highest form of user experience.

(2) Professor Donald Norman (A - Norman - Donald) from the perspective of cognitive psychology to the emotional classification. By introducing the product experience design, this paper analyzes three kinds of different types and representations of emotional experience in product design, the characteristics of each type of emotion experience, and the emphasis on the different attributes of the product. Analysis of the elements of experiential products, from the two aspects to the expression of emotional experience.

(3) According to the theory of emotional experience design, expression analysis and the corresponding principle, put forward a more complete product design process based on the emotional experience. At each stage of the process, a more concrete and feasible design method is put forward, which is used to guide the practice.

\section{References}

[1] A. Toet, M. Henselmans, M.P. Lucassen, T. Gevers, Emotional effects of dynamic textures, Iperception 2 (2011) 969-991.

[2] L. Blunt, X. Jiang, Advanced Techniques for Assessment Surface Topography,Elsevier, Sterling, U.S.A, 2003, First Edit.

[3] Y. Shin, Y. Kim, E.Y. Kim, Automatic textile image annotation by predictingemotional concepts from visual features, Image Vis. Comput. 28 (2010)526-537.

[4] S. Kim, E. Kim, K. Jeong, J. Kim, Emotion-based textile indexing using colors,texture and patterns, Lect. Notes Comput. Sci. 4292 (2006) 9-18.

[5] E. Kim, S. Kim, H. Koo, K. Jeong, J. Kim, Emotion-based textile indexing using colors and texture, Lect. Notes Comput. Sci. 3613 (2005) 1077-1080.

[6] N. Kim, Y. Shin, E. Kim, Emotion-based textile indexing usinig neural networks,Proc. 12th Int. Conf. Human-Comput. Interact. Intell. Multimodal Interact.Environ. (2007) 349-357.

[7] Q. Li, Z. Shi, S. Luo, A neural network approach for bridging the semantic gap in texture image retrieval, Proc. 2007 Int. Jt. Conf. Neural Netw. IEEE (2007)581-585.

[8] W.-Y. Lee, M.R. Luo, L.-C. Ou, Assessing the affective feelings of two- and threedimensional objects, Color Res. Appl. 34 (2009) 75-83.

[9] M.P. Lucassen, T. Gevers, A. Gijsenij, Texture affects color emotion, Color Res.Appl. 36 (2011) $426-436$.

[10] M. Solli, R. Lenz, Color emotions for multi-colored images, Color Res. Appl. 36(2011) $210-221$.

[11] X. Wang, J. Jia, Y. Wang, L. Cai, Modeling the relationship between texture semantics and textile images, Res. J. Appl. Sci. Eng. Technol. 3 (2011) 977-985.

[12] K. Reinecke, T. Yeh, L. Miratrix, R. Mardiko, Y. Zhao, J. Liu, et al., Predictingusers' first impressions of website aesthetics with a quantification of perceived visual complexity and colorfulness, ACM Press, New York, New

York, USA (2013) 2049-2058, Proc. SIGCHI Conf. Hum. Factors Comput. Syst. -CHI '13. 